Mitteilungen aus dem pharmazeutisch-chemischen Institut der Universitat Marburg.

\title{
244. Ueber das Glycocyamidin.
}

Von Erast Sobmidt.

(Eingegangen den 15. X. 1913.)

Die glatte Ueberführnng des Glycocyamins, des niedrigeren Homologen des $\mathrm{Kreat}$ ins, in Glycocyamidin, die dem Kreatinin entsprechende Base:

$$
\begin{aligned}
& \mathrm{HN}=\mathrm{C}<\underset{\text { Glycocyamin }}{\mathrm{NH}_{2}}-\mathrm{CH}_{2}-\mathrm{CO} . \mathrm{OH} \\
& \mathrm{HN}=\mathrm{C}<\mathrm{NH}-\mathrm{CH}_{2}-\mathrm{CO}
\end{aligned}
$$

war bisher mit erheblichen Schwierigkeiten verknüpft. Während das Kreatin bereits durch wiederholtes Eindampfen mit starker Salzsäure leicht quantitativ in Kreatinin übergeführt wird, kann das Glycocyamin, wie die Versuche von G. Korndörferl) lehrten, unter den gleichen Bedingungen nicht in Glycocyamidin vorwandelt werden. Auch das Erhitzen des Glycocyamins mit rauchender Salzsäure auf $140^{\circ}$ liefert in dieser Beziehung nur unbefriedigende Resultate. Das gleiche gilt nach den Beobachtungen

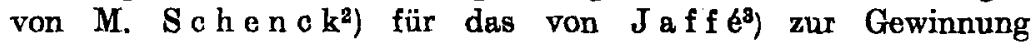
kleinerer Mengen von Glycocyamidin empfohlene Verfahren, nach welchem das Glycocyamin mit Salzsëure von $12,5 \%$ 24 Stunden lang am Rückflußkühler gekocht werden soll. G. K o r n d ö rf e r sowohl, als auch $M$. Schenck (l. c.) bereiteten daher das zur Darstellung von Methyl-Glycocyamidin verwendete Glycocyamidin nach der Methode von A. S trecker 4), indem sie trockenes Glycocyaminhydrochlorid vorsichtig auf 160 bis $170^{\circ}$ erhitzten. Da jedoch auch diese Gewinnungsweise des Glyco-

1) Dieses Archiv 1904, 630.

2) Ibidem 1910, 376.

s) Zeitschr. f. physiol. Chem. 48, 438.

4) Jahresberichte der Chemie 1861, 530. 
cyamidins mit verschiedenen Mängeln behaftet ist, infolge deren sich die Ausbeute an reiner Base ebenfalls nur zu einer mäBigen gestaltet, so schien es mir zur Fortführung der von G. K o r nd ö $\mathrm{r} f \mathrm{er}$ und von $\mathrm{M}$. S o he nck begonnenen Untersuchungen über die Isomeren des Kreatinins angezeigt zu sein, zunächst eine einfache und zugleich möglichst quantitativ verlaufende Darstellungsweise des Glycocyamidins ausfindig zu machen. Letzteres ist mir nach einigen Vorversuchen auch in so befriedigender Weise gelungen, daß das Glycocyamidin jetzt nun zu den leicht zugänglichen Verbindungen zu zählen ist.

Das zu diesen Versuchen verwendete Glycocyamin war nach dem Verfahren von $\mathrm{Nencki}$ und $\mathrm{Si}$ e b e $\mathrm{r}^{1}$ ) durch Erhitzen von Guanidinkarbonat mit Glycocoll, und zwar zum Teil unter Anwendung der von G. Kornd örfer (l. c.) empfohlenen Modifikation, dargestellt worden. Zur Ueberführung dieses Glycocyamins in Glycocyamidin wurden $5 \mathrm{~g}$ desselben in Reagenzgläsern mit so viel reiner konzentrierter Schwefelsäure versetzt, daß letztere das Glycocyamin, nachdem sio dasselbe durchdrungen hatte, noch eben bedeckte. Da hierbei eine beträchtliche Wärmeentwickelung eintritt, so geht hierdurch bereits ein großer Teil des angewendeten Glycocyamins direkt in Lösung. Das Gemisch wurde alsdann unter zeitweiligem Umrühren im Dampfbade erhitzt, wodurch alsbald eine klare, bräunlichgelb gefärbte Flüssigkeit resultierte. Da die Färbung dieser Lösung sich beim weiteren Erhitzen im Dampfbado nicht veränderte, so wurde letzteres auf 24 Stunden ausgedebnt, obschon wohl anzunehmen war, daß bereits eine weit kürzere Zeit des Erhitzens zur Ueberführung des Glycocyamins in Glycocyamidin ausreichend ist. Die einzelnen Reaktionsprodukte wurden hierauf vereinigt, mit Wasser verdünnt, die Lösung alsdann mit Natronlauge schwach alkalisch und hierauf sofort mit Essigsäure wieder schwach sauer gemacht und sohlieBlich mit Quecksilberchloridlösung und Natriumacetat in solcher Menge versetzt, daß direkt keine weitere Ausscheidung von Glycocyamidin-Quecksilberchlorid erfolgte. Der hierdurch erzeugte, rein weißgefärbte Niederschlag wurde alsdann nach kurzem Absetzen auf einem Saugfilter gesammelt und mit Wasser sorgfältig ausgewaschen. Hierauf wurde der Niederschlag in Wasser gleichmäßig suspendiert und unter Anwendung von mäßiger Wärme durch Einleiten von Schwefelwasserstoff zerlegt. Die von dem gleichmäßig schwarz gefärbten Niederschlage durch Absaugen und Auswaschen getrennte,

1) Journal für praktische Chemie. Neue Folge 17, 477. 
vollständig farblose Flüssigkeit wurde aledann zur Trockne verdampft und der Rückstand aus siedendem Alkohol umkrystallisiert.

$20 \mathrm{~g}$ Glycocyamin lieferten unter obigen Bedingungen, bei sorgfältiger Aufarbeitung der Mutterlaugen, 19 bis $20 \mathrm{~g}$ reines Glycooyamidinhydrochlorid. Letzteres bildete kleine, farblose, zu Drusen gruppierte Nadeln, welche sich gegen $200^{\circ}$ bräunten und bei 208 bis $210^{\circ}$ unter Schwärzung schmolzen.

$0,370 \mathrm{~g}$ verloren bei $100^{\circ} 0,0086 \mathrm{~g}=2,32 \%$ an Gewicht. $0,3614 \mathrm{~g}$ getrocknote Substanz lieforten $0,3794 \mathrm{~g} \mathrm{AgCl}$.

Gefunden: $\mathrm{HCl} 26,70$

Berechnet für $\mathrm{C}_{3} \mathrm{H}_{3} \mathrm{~N}_{3} \mathrm{O}, \mathrm{HCl}$ : 26,90

Zur Gewinnung des freien Glycocyamidins wurde die durch Erhitzen von Glycocyamin mit Schwefelsäure erhaltene Lösung mit Wasser verdünnt, hierauf mit alkalifreier Baryumhydroxydlösung bis zur schwach sauren Reaktion versetzt und schlieBlich durch Digestion mit frisch gefälltem Baryumkarbonat vollständig von Schwefelsäure befreit. Die filtrierte Flüssigkeit wurde alsdann zur Trockne verdunstet, der Rückstand in siedendem Alkohol, unter Zusatz von wenig Wasser, gelöst und die Lösung hierauf der freiwilligen Verdunstung überlassen. Das Glycocyamidin resultierte auf diese Weise in farblosen, kleinen, meist zu Krusten vereinigten Blättchen von schwach alkalischer Reaktion. Bei $220^{\circ}$ fängt das Glycocyamidin an sich «u schwärzen, ohne jedoch bei $250^{\circ} \mathrm{zu}$ schmelzen.

\section{Silberverbindungen des Glycocyamidins.}

Die Silberverbindungen des Glycocyamidins sind bereits von G. Korndörfer (l. c.) studiert worden. Ich habe dieselben beiläufig von neuem untersucht, da ich das Glycocyamidinsilber zur Darstellung von Alkyl-Glyeocyamidinen als Ausgangsmaterial verwenden wollte.

Versetzt man eine verdünnte wässerige Lösung des Glycocyamidins mit Silbernitratlösung, so tritt keine oder doch nur eine geringe Trübung ein. Fügt man jedoch vorsichtig Ammoniak zu dieser Mischung, o entsteht ein voluminöser, ziemlich lichtempfindlicher, weißer Niederschlag, der beim Stehen im Dunkeln allmählich fein krystallinische Beschaffenheit annimmt. In Salpetersäure ist dieser Niederschlag leicht löslich; auch von überschüssiger Ammoniakflüssigkeit wird derselbe, namentlich beim gelinden Erwärmen, gelöst. 
Sobald dieser Niederschlag krystallinische Beschaffenheit angenommen hatte, wurde derselbe gesammelt, scharf abgesogen und mit kleinen Mengen Wasser sorgfältig ausgewaschen. Nach dem Abpressen zwischen Tonplatten und Trocknen bei gewöhnlicher Temperatur entsprach die Zusammensetzung dieses Produkts der Formel $\mathrm{C}_{3} \mathrm{H}_{4} \mathrm{~N}_{3} \mathrm{OAg}+\mathrm{H}_{2} \mathrm{O}$. Präparate versohiedener Darstellung ergaben bei der Analyse folgende Werte:

1. $0,4464 \mathrm{~g}$ verloren bei $100^{\circ} 0,0352 \mathrm{~g}$ an Gewicht und lieferten $0,2838 \mathrm{~g} \mathrm{AgCl}$.

2. $0,2244 \mathrm{~g}$ verloren bei $100^{\circ} 0,0170 \mathrm{~g}$ an Gewicht und lieferten $0,1084 \mathrm{~g} \mathrm{Ag}$.

3. $0,2234 \mathrm{~g}$ der bei $100^{\circ}$ getrockneten Substanz lieferten $0,1164 \mathrm{~g} \mathrm{Ag}$.

Gefunden:

Berechnet für

1. 2 .

$\mathrm{H}_{2} \mathrm{O} \quad 7,89 \quad 7,58$

Gefunden:

1. $2 . \quad 3$.

Ag $\quad 51,94 \quad 52,26 \quad 52,10$

$\mathrm{C}_{3} \mathrm{H}_{4} \mathrm{~N}_{3} \mathrm{OAg}+\mathrm{H}_{2} \mathrm{O}$ :

8,03

Berechnet für

$\mathrm{C}_{3} \mathrm{H}_{4} \mathrm{~N}_{3} \mathrm{OAg}$ :

52,37

G. Korndörf e r (l. c.) erhielt unter ähnlichen Versuchsbedingungen ein Glycocyamidinsilber derselben Zusammensetzung, jedoch verlor dasselbe bei $100^{\circ}$ nicht an Gewicht.

Das Filtrat der obigen Silberverbindung lieferte nach dem Ansäuern mit Salpetersäure und darauffolgendem Verdunsten lange, lockere, seidenglänzende Nadeln, welche bei $100^{\circ}$ nicht an Gewicht verloren. Diese Verbindung enthielt, im Gegensatz zu der im vorstehenden beschriebenen, Salpetersäure.

Produkte verschiedener Darstellung ergaben bei der Analyse folgende Werte:

1. $0,2732 \mathrm{~g}$ enthielten $0,0782 \mathrm{~g}$ Ag.

2. $0,1469 \mathrm{~g}$ enthielten $0,0419 \mathrm{~g} \mathrm{Ag}$.

3. $0,2795 \mathrm{~g}$ enthielten $0,0797 \mathrm{~g} \mathrm{Ag}$.
Gefunden:
Berechnet für
1. $2 . \quad 3$.
$\left(\mathrm{C}_{3} \mathrm{H}_{5} \mathrm{~N}_{3} \mathrm{O}\right)_{2} \mathrm{AgNO}_{3}$ :

Ag $28,63 \quad 28,50 \quad 28,52 \quad 28,31$

Eine Verbindung von dem gleichen Silbergehalt $(28,56 \%)$ erhielt G. K o r n d ö r f e r (l. c.) als krystallinischen Niedersohlag beim direkten Zusammenbringen von konzentrierter wässeriger Glycocyamidin- und Silbernitratlösung und darauffolgendem Reiben der Gefäßwände mit einem Glasstab.

(Schlub folgt.) 
Neberı diesen lockercn, seideglänzenden Nadeln resultierten aus den letzten; sehr konzentrierten Mutterlaugen noch kleine, durchsichtige, zu Drusen gruppierte Krystalle, deren Menge sich đureh Zusstz von absolutem Alkohol noch vermehrte. Diese Ausscheidungen enthielten ebenfalls Salpetersäure. Bei $100^{\circ}$ verloren dieselben nicht an Gewicht.

1. 0,4212 g lieferten 0,2234 g AgCl.

2. $0,1364 \mathrm{~g}$ lieferten $0,0552 \mathrm{~g}$ Ag.

\begin{tabular}{cccc} 
& \multicolumn{2}{c}{ Gefunden: } & Berechnet für \\
& 1. & 2. & $\mathrm{C}_{3} \mathrm{H}_{8} \mathrm{~N}_{8} \mathrm{O}, \mathrm{AgNO}_{3}:$ \\
$\mathrm{Ag}$ & $\mathbf{3 9 , 9 2}$ & $\mathbf{4 0 , 4 8}$ & $\mathbf{4 0 , 1 0}$
\end{tabular}

Glycocyamidinplatinchlorid: $\left(\mathrm{C}_{3} \mathrm{H}_{5} \mathrm{~N}_{3} \mathrm{O}, \mathrm{HCl}\right)_{2}$ $\mathrm{PtCl}_{4}+2 \mathrm{H}_{2} \mathrm{O}$, ist bereits von $\mathrm{Str}$ e o k e (l. c.) dargestelt und als in Nadeln krystallisierend beschrieben worden. Das zum Vergleich mit den Platindoppelsalzen der Methylierungsprodukte des Glycocyamidins von mir dargestellte Glycocyamidinplatinchlorid bildete bei langsamer Verdunstung kompakte, leicht lösliche, rotgelbe Säulen oder große, wohl ausgebildete, durchsichtige, anscheinend monokline Tafeln. Beide Formen entsprachen in ihrer Zusammensetzung der von $\mathrm{Strecker}$ angegebenen Formel $\left(\mathrm{C}_{3} \mathrm{H}_{5} \mathrm{~N}_{3} \mathrm{O}, \mathrm{HCl}\right)_{2} \mathrm{PtCl}_{4}+2 \mathrm{H}_{2} \mathrm{O}$.

Das entwässerte Doppelsalz schmolz bei $260^{\circ}$ noch nicht, jedoch trat vorher bereits Sintern und Schwarzfärbung ein.

1. $0,3843 \mathrm{~g}$ der sëulenförmigen Krystalle verloren bei $100^{\circ}$ $0,021 \mathrm{~g}$ an Gewicht; $0,2535 \mathrm{~g}$ wasserfreies Salz enthielten $0,0738 \mathrm{~g} \mathrm{Pt}$.

2. $0,426 \mathrm{~g}$ der tafelförmigen Krystalle verloren bei $100^{\circ} 0,0246 \mathrm{~g}$ an Gewicht; 0,3971 $\mathrm{g}$ wasserfreies Salz enthielten 0,1264 $\mathrm{g}$ Pt.

\begin{tabular}{cccc} 
& \multicolumn{2}{c}{ Gefunden: } & Berechnet für \\
& 1. & 2. & $\left(\mathrm{C}_{3} \mathrm{H}_{3} \mathrm{~N}_{3} \mathrm{O}, \mathrm{HCl}\right)_{2} \mathrm{PtCl}_{4}+2 \mathrm{H}_{2} \mathrm{O}:$ \\
$\mathrm{H}_{2} \mathrm{O}$ & $\mathbf{5 , 4 5}$ & 5,77 & 5,59 \\
& Gefunden: & Berechnet für \\
& 1. & 2. & $\left(\mathrm{C}_{3} \mathrm{H}_{6} \mathrm{~N}_{8} \mathrm{O}, \mathrm{HCl}\right)_{2} \mathrm{PtCl}_{4}:$ \\
Pt & 31,61 & 31,83 & 32,03
\end{tabular}

Glycocyamidingold ch lorid. Die Zusammensetzung des in Wasser sehr leicht löslichen und leicht zersetzlichen Glycocyamidinaurats scheint je nach den obwaltenden Versuchsbedingungen eine verschiedene zu sein. G. Kornd ö r f e r (l. c.)

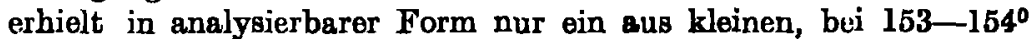
schmelzenden Blättchen bestehendes Aurat der Formel $\mathrm{C}_{3} \mathrm{H}_{5} \mathrm{~N}_{8} \mathrm{O}$, $\mathrm{AuCl}_{3}$, vermochte dagegen ein normal zusammengesetztes Aurat $\mathrm{C}_{8} \mathrm{H}_{6} \mathrm{~N}_{2} \mathrm{O}, \mathrm{HCl}+\mathrm{AuCl}_{3}$ im reinen Zustande nioht $\mathrm{zu}$ isolieren. 
Letzteres Doppelsalz wird jedoch ohne erhebliche Schwierigkeiten orhalten, wenn man eine konzentrierte wä日serige Lösung von Glycocyamidinhydrochlorid mit einem beträchtlichen Ueberschuß von Goldchloridchlorwasserstofflösung versetzat und das Gemisch hierauf über Aetzkalk verdunsten-tuBt: Es scheiden sich dann allmählich wohlausgebildete, durchsichtige, tafelförmige Krystalle in betrüohtlioher Größe aus, die sich leicht von der Mutterlauge und den mitausgeschiedenen Goldflitterchen trennen lassen. Diese, in Wasser sehr leicht löslichen Krystalle schmelzen bei $158^{\circ}$. Die Analyse von Produkten verschiedener Darstellung ergab folgende Daten:

1. $0,210 \mathrm{~g}$ enthielten $0,094 \mathrm{~g} \mathrm{Au}$.

2. $0,4278 \mathrm{~g}$ enthielten $0,180 \mathrm{~g} \mathrm{Au}$ und lieferten 0,5482 $\mathrm{g} \mathrm{AgCl}$. Gefunden:

1. 2.

Au $\quad 44,63 \quad 44,41$

$\mathrm{Cl} \quad-\quad 31,70$
Berechnet für $\mathrm{C}_{3} \mathrm{H}_{5} \mathrm{~N}_{8} \mathrm{O}, \mathrm{HCl}, \mathrm{AuCl}_{8}$ :

44,90

32,33

Ueber das Verhalten des Glycocyanidinsilbers gegen Jodmethyl und gegen Dinethylsulfat werde ioh in Finer weiteren Abhandlung berichten.

\section{Aus der medizinischen Abteilung des Universitatslaboratoriums Freiburg i Dr. \\ Ueber Digitoxin und Gitalin.}

Von H. Kiliani.

(Eingegangen den 18. XI. 1013.)

In einer fur die Digitalischemie wichtig gewordenen Árbeit) hat $\mathrm{Kr}$ aft ein neues wirksames, aber amorphes Glykosid, das G it a lin, beschrieben, welches sich von allen früher bekannten einschlägigen Stoffen durch eine ganz außergewöhnliche Labilität seines Moleküls und vom ,,wirklichen" Digitoxin durch die Bildung eines krystallisierten Hydrats unterscheiden soll; er glaubt ferner, aus seinen Beobachtungen, ,den Schluß ziehen zu dürfen, daß K i l i a n i's Digitoxin noch nicht ganz rein war, sondern einen ziemlichen Mitanteil des damals noch unbekannten Gitalins enthielt"; das

1) Dieses Archiv 250, 118. 